\title{
Prevalencia de bacteriemias relacionadas con el catéter de hemodiálisis en una unidad hospitalaria
}

\author{
Patricia Arribas Cobo
}

Graduada en Enfermería. Hospital Universitario Infanta Leonor. Madrid

\section{Resumen}

En la actualidad ha aumentado el uso de catéteres tunelizados como acceso vascular permanente para los pacientes en hemodiálisis a pesar de las complicaciones que tiene asociadas, como puede ser la bacteriemia relacionada con el catéter.

El objetivo de este trabajo es mostrar la tasa de prevalencia de bacteriemia relacionada con el catéter para hemodiálisis, obtenida con la aplicación estricta de un protocolo de cuidados del catéter tunelizado por un personal bien entrenado en la unidad de hemodiálisis del Hospital Universitario Infanta Leonor entre abril de 2008 y abril de 2013, así como describir las posibles causas de bacteriemia relacionada con el catéter, la relación con alguna de las variables y su evolución en los últimos años.

Durante los 5 años de estudio tuvimos 9 episodios de bacteriemia relacionada con el catéter, lo que supone una tasa global de prevalencia de 0,25/1000 días de catéter. La tasa por cada uno de los años fue menor de $1 / 1000$ días de catéter.

Los pacientes que tuvieron una bacteriemia relacionada con el catéter tuvieron implantados mayor número de catéteres que los que no la tuvieron, siendo esta diferencia significativa $(p=0,027)$.

No se encontraron diferencias con respecto a la edad, el sexo, la diabetes mellitus, el índice de co-

\section{Correspondencia: \\ Patricia Arribas Cobo \\ C/ Florencia 8, $1^{\circ} \mathrm{A}$ \\ 28030 Madrid \\ E-mail: patriarribas@gmail.com}

morbilidad de Charlson y la localización de los catéteres entre los pacientes que padecieron una bacteriemia relacionada con el catéter de los que no.

\section{PALABRAS CLAVE:}

- BACTERIEMIA

- ACCESO VASCULAR

- CATÉTER, HEMODIÁLISIS

\section{Prevalence of catheter-related bacteraemia in a hospital haemodialysis unit}

\section{Abstract}

Currently has increased the use of tunneled catheters as permanent vascular access for hemodialysis patients despite having associated complications, such as bacteremia.

The aim of this work is to show the incidence of catheter-related bacteremia for hemodialysis obtained with the strict implementation of a protocol tunneled catheter care by well-trained personnel in the Hemodialysis Unit of University Hospital Infanta Leonor between April 2008 and April 2013, and to describe possible causes of bacteremia, the relationship with any of the variables and their evolution in recent years.

During 5 years of study there had been 9 episodes of catheter-related bacteremia which is an overall incidence rate of 0.25 / 1000 catheter days. The fee for each of the years was less than $1 / 1000$ catheter days.

Patients who had bacteremia had catheters implanted more than those who had not, and this difference was significant $(p=0.027)$. 
No differences were found with respect to age, sex, diabetes mellitus, the Charlson comorbidity index and location of catheters among patients who developed bacteremia from those without.

\section{KEY WORDS:}

BACTEREMIA

- VASCULAR ACCESS

TUNNELED CATHETER

- HEMODIALYSIS

\section{Introducción}

La prevalencia actual de los pacientes en tratamiento renal sustitutivo (TRS) es de 1078 casos por millón de habitantes, estando el $50,12 \%$ de los pacientes con un trasplante renal funcionante (TR), el $44,61 \%$ en programa de hemodiálisis (HD) y el $5,27 \%$ en programa de diálisis peritoneal (DP) ${ }^{1}$.

Las causas más importantes de mortalidad de los pacientes en HD continúan siendo las causas cardiovasculares, seguido de las infecciones ${ }^{1}$.

El empleo de catéteres tunelizados (CT) en las venas centrales constituye una alternativa de acceso vascular permanente, ya que proporciona un acceso al torrente circulatorio de forma rápida y permite la realización de una diálisis eficaz, pero no debe considerarse como primera opción, ya que los otros accesos vasculares ofrecen mejores resultados y menor grado de complicaciones ${ }^{2}$. Por lo tanto, deberán ser utilizados sólo en aquellos pacientes en los que no sea posible el uso de una fístula arteriovenosa autóloga o prótesis arteriovenosa, ya sea por imposibilidad de creación (por ausencia de arterias con un flujo adecuado) o en espera de desarrollo adecuado ${ }^{3}$.

El problema es que el uso de CT como acceso vascular para HD en comparación con la fístula autóloga, se asocia de forma independiente a una mayor comorbilidad inicial del paciente, a una mayor mortalidad tanto al inicio de la diálisis como a lo largo del seguimiento, y se asocia a que dicha mortalidad sea más elevada en los pacientes que permanecen más tiempo con un $\mathrm{CT}^{4}$.

Para la manipulación de las conexiones del CT, conexión al circuito de diálisis y sellado al final, se recomienda efectuar un lavado higiénico de las manos y utilizar campo y guantes estériles. $Y$ se recomienda que tanto el paciente como el personal que lo manipule usen mascarilla ${ }^{3}$.

Los CT, pese a las recomendaciones que dan todas las sociedades nefrológicas constituyen un porcentaje elevadísimo de los accesos vasculares en $\mathrm{HD}^{5}$. Así, en el estudio DOPPS (Dialysis Outcomes and Practice Patterns Study) en su edición cuatro (2010-2012), indica que el uso de CT para HD en España se sitúa en un 30\% en el uso de CT para HD 6 .

Por otro lado, en nuestra Comunidad Autónoma, se muestra que el $44,6 \%$ de los pacientes inician HD con un catéter, que el CT es el acceso utilizado en el $29,5 \%$ de los pacientes ya prevalentes en HD, y que en los últimos años incluso se ha visto incrementado su uso ${ }^{7}$. Este porcentaje es mucho más elevado de lo que recomienda la Sociedad Española de Nefrología, en cuyos indicadores de seguimiento y cuidado de accesos vasculares plantea el objetivo de tener menos de un $10 \%$ de pacientes dializados en la unidad a través de un $\mathrm{CT}^{8}$.

La utilización de CT se asocia a tres problemas fundamentales como son la trombosis, la disfunción y las infecciones. Tanto la trombosis como la disfunción requieren actuaciones por parte del personal (recolocación del catéter, lavados enérgicos con suero fisiológico, pautas de fibrinolisis local, etc.) que aumentan a su vez el riesgo de padecer una infección.

Aunque la infección es menos frecuente, la bacteriemia relacionada con el catéter (BRC) causa una elevada morbimortalidad.

Los microorganismos responsables de una de las dos terceras partes de las BRC son grampositivos. Staphylococcus aureus y los Staphylococcus coagulasa negativos. El Staphylococcus aureus es un microorganismo muy virulento capaz de ocasionar complicaciones metastásicas?.

Beathard y Urbanes ${ }^{10}$ plantean unos rangos de tasas de BRC para que podamos valorar cómo es nuestra práctica asistencial. Así, tasas por debajo de 1 evento/1000 días de catéter lo caracterizan de excelente, entre 1 y 2 eventos sería una buena práctica. Pero según estos autores, cualquier centro que tenga una tasa mayor a 2 eventos/1000 días-catéter debería plantearse mejorar sus prácticas de prevención y tasas por encima de 6-7 sería una mala práctica.

La frecuencia de BRC es variable y oscila entre $0,7^{11}$ a 9,1 ${ }^{12}$ eventos/1000 días-catéter según distintos estu- 
dios internacionales. La tasa de BRC en las unidades españolas también es variable y oscila entre $0,24{ }^{13} \mathrm{a}$ $5,47^{14}$ eventos/1000 días-catéter. Aunque bien es cierto que se publica muy poco sobre las tasas de BRC que se tienen en distintas unidades, este amplio rango refleja importantes diferencias en la práctica clínica.

En la mayoría de las publicaciones de los últimos años estas prácticas se basan en medidas farmacológicas como antibióticos a nivel de la luz del catéter o del orificio de salida del mismo, aunque los efectos a largo plazo, como la aparición de resistencias bacterianas, nefrotoxicidad u ototoxicidad ${ }^{15}$ según el fármaco utilizado, se desconocen ${ }^{16}$. Otras medidas farmacológicas que se han utilizado ha sido el uso de un antimicrobiano unido a un agente anticoagulante, algo que, si bien reduce los riesgos de formación de resistencias como los antibióticos, supone un incremento elevado del coste del tratamiento ${ }^{19}$. También se han estudiado medidas de mejora con el uso de bioconectores ${ }^{13,17}$.

El objetivo de este trabajo es mostrar la tasa de prevalencia de bacteriemias relacionadas con el catéter tunelizado para HD obtenida con la aplicación estricta de un protocolo de cuidados de CT por un personal bien entrenado en una Unidad de HD.

Como objetivo secundario se pretende describir las posibles causas de BRC, la relación con alguna de las variables y su evolución en los últimos años.

\section{Material y métodos}

Se trata de un estudio de serie de casos, donde se recogieron retrospectivamente todos los datos relacionados con la prevalencia de BRC en una población de HD desde la apertura de la Unidad en abril de 2008 hasta abril de 2013 (5 años de seguimiento). Durante todo este tiempo se ha usado un protocolo específico de profilaxis de infección.

La muestra la constituyen 100 pacientes, que se dializaron en algún momento a través de un CT. Los criterios de exclusión fueron: pacientes que se dializaron a través de un catéter no tunelizado o pacientes que se dializaron en nuestra unidad de forma transeúnte por estar de vacaciones.

Las variables se recogieron de las historias clínicas de los pacientes y fueron: edad (a la fecha de inserción del catéter), sexo, diabetes mellitus, fecha de inserción y retirada del catéter (para calcular tiempo efectivo), Índice de comorbilidad de Charlson, número de catéteres insertados a cada paciente, localización del catéter, número de BRC y germen causante.

Para los casos de BRC se recogió si en los 15 días previos a la aparición de la BRC el paciente había estado dializándose en otra unidad, si hubiera tenido disfunción del catéter o si hubiera tenido signos de infección del orifico de salida del catéter como aparición de exudado costra o enrojecimiento del mismo.

Las enfermeras a su incorporación en la Unidad siguen un periodo de formación de alrededor de un mes. Cada enfermera dializa habitualmente un máximo de 4 pacientes, en una unidad con 2 turnos de HD distribuidos en mañana y tarde. Al finalizar cada turno se procede a limpiar exhaustivamente la sala y los monitores.

La BRC se definió como la aparición de fiebre durante la sesión de diálisis en un paciente con CT en el que no hubiera otra explicación etiológica para la infección.

La tasa de BRC se calculó como $\mathrm{n}^{\circ} \mathrm{BRC} \times 1000 /$ días de seguimiento. Se usó el programa Excel para la recogida y el cálculo de resultados de las tasas de BRC que vienen expresados como mediana (rango).

Se utilizó la t-Student para relacionar la BRC con las variables cuantitativas y el Chi-cuadrado para relacionarlas variables cualitativas. Para ello se utilizó el programa SPSS 15 para Windows.

\section{Resultados}

Durante el periodo analizado se utilizaron un total de 139 CT en 100 pacientes; 35 mujeres (35\%) y 65 hombres $(65 \%)$, con una edad media a la fecha de la inserción del catéter de 65,1 con un rango entre 18 y 90 años $y$ de los que el $43 \%$ eran diabéticos.

El índice de comorbilidad de Charlson de los pacientes fue de media 7,23 (DE: 2,79), con un rango de entre 2 y 15.

De los 100 pacientes, 25 de ellos tuvieron más de 1 catéter. Las causas de los recambios fueron: en 7 ocasiones por BRC, 1 por tunelitis, 1 por extrusión del cuff, 1 por salida accidental del mismo, 4 por deterioro o rotura de alguna de las ramas del catéter y 25 por disfunción. 
La localización de los catéteres fue la que se muestra en la Fig. 1.

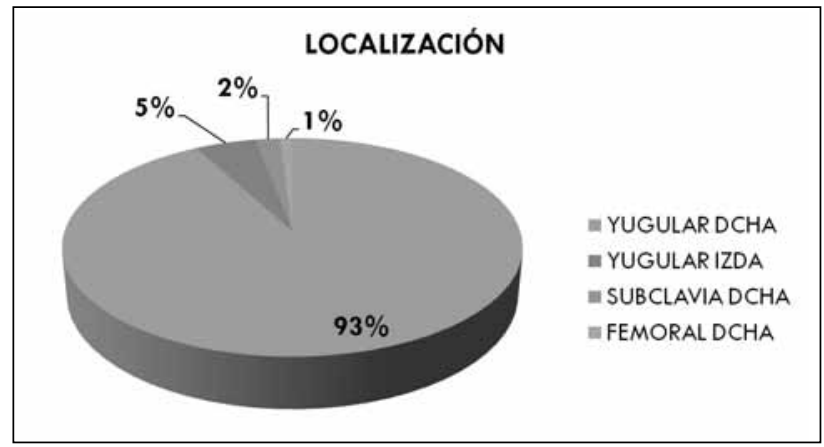

Fig. 1. Localización de los catéteres.

El tiempo total de seguimiento de los 5 años fue de 36.183 días, con una mediana de 206 (rango intercuartílico: 95-413) días por paciente, teniendo en cuenta que 1 de los pacientes ha estado durante todo el tiempo de estudio (1.769 días) y el paciente que menos días tuvo puesto un catéter fueron 12 días.

Durante este periodo ocurrieron 9 episodios de BRC lo que supone una tasa de prevalencia en los cinco años de seguimiento de 0,25/1000 días de catéter.

Los resultados que observamos al analizar la tasa por cada año para valorar la evolución a lo largo del tiempo nos dejan ver que en ninguno de los años se llega a 1 evento por cada 1000 días de catéter y se muestran en la Tabla 1.

\begin{tabular}{|c|c|c|c|c|c|c|c|}
\hline AÑO & $2008 *$ & 2009 & 2010 & 2011 & 2012 & $2013 * *$ & TOTAL \\
\hline $\mathbf{T}^{0}$ & 2.048 & 6.533 & 7.113 & 7.744 & 7.810 & 2.079 & 36.183 \\
\hline $\mathbf{N}^{0}$ BRC & 1 & 1 & 0 & 2 & 3 & 2 & 9 \\
\hline TASA & 0,48 & 0,15 & 0 & 0,26 & 0,38 & 0,96 & 0,25 \\
\hline
\end{tabular}

Tabla 1. Tasa de bacteriemias por cada año de seguimiento.

* Desde abril hasta diciembre de 2008.

** Desde enero hasta abril de 2013.

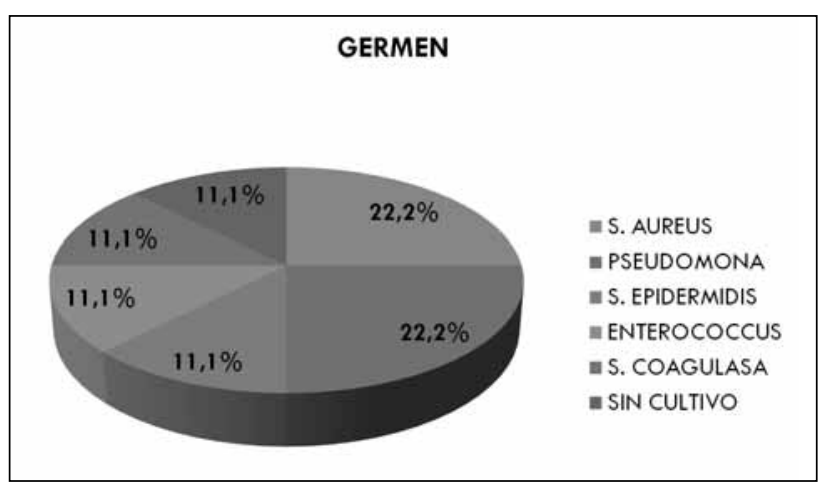

Fig. 2. Tipos de germen causantes de las BRC.
Dos de los pacientes que tuvieron una BRC fallecieron por esta causa, lo que supone una mortalidad de un $22,2 \%$.

Los gérmenes causantes se muestran en la Fig. 2.

Estudiando solo los casos de BRC encontramos que durante los quince días previos a la aparición de la BRC, la distribución de las eventualidades es la que se muestra en la Fig. 3.

Los resultados con respecto a la localización del catéter,

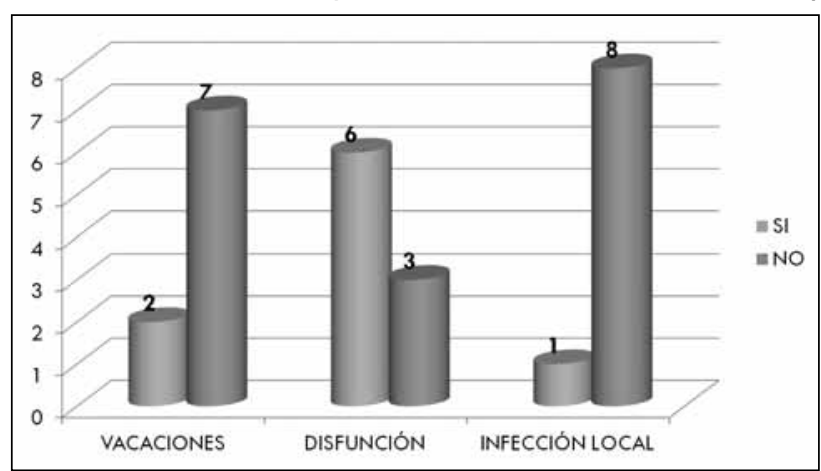

Fig. 3. Eventualidades de los casos de BRC días previos a la misma.

el sexo, la diabetes, el índice de comorbilidad de Charlson, la edad y el número de catéteres se muestran en las siguientes tablas: Tablas 2, 3, 4, 5, 6 y 7.

\begin{tabular}{|c|c|c|c|c|}
\hline $\begin{array}{c}\text { LOCALI- } \\
\text { ZACIÓN }\end{array}$ & YUG DCHA & YUG IZDA & $\begin{array}{c}\text { SUBC } \\
\text { DCHA }\end{array}$ & $\begin{array}{c}\text { FEM } \\
\text { DCHA }\end{array}$ \\
\hline SI BRC & $82,8 \%$ & $0 \%$ & $11,1 \%$ & $11,1 \%$ \\
\hline NO BRC & $93,4 \%$ & $5,5 \%$ & $1,1 \%$ & $0 \%$ \\
\hline
\end{tabular}

Tabla 2. Relación entre los casos de BRC y los que no según localización. $p=0,203$

\begin{tabular}{|c|c|c|}
\hline SEXO & HOMBRES & MUJERES \\
\hline SI BRC & $55,6 \%$ & $44,4 \%$ \\
\hline NO BRC & $65,9 \%$ & $34,1 \%$ \\
\hline
\end{tabular}

Tabla 3. Relación entre los casos de BRC y los que no según sexo. $p=0,716$

\begin{tabular}{|c|c|c|}
\hline $\begin{array}{c}\text { DIABETES } \\
\text { MELLITUS }\end{array}$ & SI & NO \\
\hline SI BRC & $55,6 \%$ & $44,4 \%$ \\
\hline NO BRC & $41,8 \%$ & $58,2 \%$ \\
\hline
\end{tabular}

Tabla 4. Relación entre los casos de BRC y los que no según diabetes. $p=0,493$

\begin{tabular}{|c|c|}
\hline I. C. CHARLSON & MEDIA \\
\hline SI BRC & $6,44 \%$ \\
\hline NO BRC & $7,3 \%$ \\
\hline
\end{tabular}

Tabla 5. Relación entre los casos de BRC y los que no según el índice de comorbilidad de Charlson. $p=0,384$ 


\begin{tabular}{|c|c|}
\hline EDAD & MEDIA \\
\hline SI BRC & 58,1 \\
\hline NO BRC & 65,8 \\
\hline
\end{tabular}

Tabla 6. Relación entre los casos de BRC y los que no según la edad. $p=0,146$

\begin{tabular}{|c|c|}
\hline $\mathbf{N}^{0}$ CATÉTERES & MEDIA \\
\hline SI BRC & 2,8 \\
\hline NO BRC & 1,3 \\
\hline
\end{tabular}

Tabla 7. Relación entre los casos de BRC y los que no según el número de catéteres de los que fueron portadores. $p=0,027$

\section{Discusión y conclusiones}

El trabajo muestra como, aplicando un protocolo estricto de asepsia de manipulación del CT para HD, se logra una tasa de BRC que se puede clasificar de excelente, ya que ni a nivel global de los cinco años, ni en cada uno de los años por separado se ha superado una tasa de 1/1000 días de catéter.

No hemos encontrado diferencias según la localización del catéter al igual que en otros estudios encontrados ${ }^{18}$, lo que además se explica porque la mayoría de los catéteres tiene la misma localización (yugular derecha).

Sólo se ha encontrado una diferencia estadísticamente significativa entre los casos de BRC y es el número de catéteres de los que han sido portadores, siendo mayor en los pacientes que habían sufrido una BRC. Esta diferencia, aunque tiene un valor significativo no lo es tanto clínicamente, ya que una de las posibles indicaciones tras la aparición de una BRC es el cambio del catéter.

El personal de enfermería tiene un papel primordial en la prevención de la BRC. La formación inicial y continuada, así como la adecuación numérica del personal al número y complejidad de los pacientes, son elementos fundamentales para la prevención de las infecciones por catéter y otras complicaciones.

La relación coste-efectividad, viene determinada por la diferencia entre el uso de material fungible, el ratio enfermera/auxiliar de enfermería-paciente y la formación continuada del personal de enfermería con respecto al tratamiento y repercusiones de aumentar la morbilidad de los pacientes, en este caso de sufrir una BRC.

No se encontraron diferencias con respecto al resto de variables del estudio; la edad, el sexo, la diabetes mellitus, el índice de comorbilidad de Charlson y la localiza- ción del catéter entre los pacientes que padecieron una $B R C$ de los que no.

En toda unidad de HD deberían existir protocolos escritos de todos los procedimientos relacionados con la manipulación de los CT y debería realizarse una evaluación periódica de su seguimiento, con el fin de evitar su incumplimiento con el paso del tiempo. Así mismo, considero que se deberían introducir indicadores para medir la calidad de los cuidados que prestamos a nuestros pacientes y conocerlos para también poder medirnos y compararnos con otras unidades y poder valorar nuestra práctica y su evolución a lo largo del tiempo.

También es importante, dada la relevancia en la morbimortalidad, el análisis de cada una de las infecciones, buscando el origen y aplicando medidas correctoras.

Las limitaciones de este trabajo son:

- Las variables se han recogido de manera retrospectiva de las historias clínicas, por lo que puede haber alguna deficiencia en los datos.

- Se deberían haber recogido más variables, como signos de infección del orificio de salida o aparición de tunelitis en todos los casos y no solo en los pacientes de BRC.

- No se ha podido recoger si los pacientes que padecieron BRC habían sido portadores de catéter durante más tiempo.

Todas estas variables se deberían incluir como futuras líneas de investigación.

\section{Agradecimientos}

- A Nunci por su aliento constante en el progreso de enfermería y en el mío personal.

- A Marta Albalate por sugerir la idea y por su disposición continua en la colaboración con la enfermería.

- A todo el personal de enfermería que realiza la labor diaria.

- A los pacientes de la unidad de diálisis, para los cuales va dedicado con todo mi cariño mi trabajo de cada día. 
Recibido: 30 Agosto 2013

Revisado: 10 Septiembre 2013

Modificado: 10 Septiembre 2013

Aceptado: 12 Septiembre 2013

\section{Bibliografía}

1. Registros de la SEN. Informe de diálisis y trasplante.

2. http://www.senefro.org/modules/webstructure/ files/reercongsen2012.pdf.

3. Fernández -Gallego J, López V, Martín MA, Toledo R. El catéter venoso central crónico tunelizado aumenta la mortalidad en hemodiálisis. Nefrología 2005: 25 (6): 720.

4. Rodríguez Hernández JA, Gutiérrez Julián JM, et al. Guías de acceso vascular en hemodiálisis. Nefrología (Guías SEN) 2005; 25 (Suplemento I): 64-92.

5. Gruss E, Portolés J, et al. Repercusiones clínicas y económicas del uso de catéteres tunelizados de hemodiálisis en un área sanitaria Nefrología 2009;29 (2):123-129.

6. Tordoir J, Canaud B, Haage P, Konner K, Basci A, Fouque D, et al. EBPG on vascular access. Nephrol Dial Transplant 2007: 22: 88-117.

7. Informe anual. DOPPS (Dialysis Outcomes and Practice Patterns Study) 4 (2010). http://www. dopps.org.

8. Gruss $E$, Portolés $J$, et al. Los modelos de atención al acceso vascular condicionan resultados heterogéneos en los centros de una misma comunidad. Nefrología 2010; 30 (3):310-6.

9. Arenas MD, Álvarez-Ude F et al. Impacto del seguimiento de indicadores de calidad en hemodiálisis. Nefrología 2004: 24 (3): 261-275.

10. Aguinaga A, del Pozo J.L Infección asociada a catéter en hemodiálisis: diagnóstico, tratamiento y prevención. NefroPlus 2011; 4(2):1-10.

11. Beathard $G A$, Urbanes $A$. Infection associated with tunneled hemodialysis catheter. Semin Dial 2008; 21:528-538.

12. Power A, Duncan N, Singh SK, Brown W, Dalby E, Edwards $C$, et al. Sodium citrate versus heparin catheter locks for cuffed central venous catheters: a single-center randomized controlled trial. Am J Kidney Dis 2009; 53: 1034-1041.

13. Feely T, Copley A, Bleyer AJ. Catheter lock solutions to prevent bloodstream infections in high-risk hemodialysis patients. Am J Nephrol 2007; 27: 2429.

14. Albalate $M$, Pérez García $R$, de Sequera $P$, Alcázar R, Puerta $M$, Ortega $M$, Mossé $A$, Crespo $E$. ¿Hemos olvidado lo más importante para prevenir las bacteriemias en pacientes portadores de catéteres para hemodiálisis? Nefrología 2010; 30 (5):573-577.

15. Cobo Sánchez JL, Sáinz Alonso RA et al. Estudio comparativo de la incidencia de bacteriemias relacionadas con el catéter de hemodiálisis: bioconectores frente a conexión directa. Rev Esp Enferm Nefrol 2011; 14 (4): 230-235.

16. Dogra $G$, Herson $H$, Hutchinson $B$, Irish $A$, Heath $C$, Golledge $C$, et al. Prevention of tunneled hemodialysis catheter-related infections using catheterrestricted filling with gentamicin and citrate: a randomized controlled study. J Am Soc Nephrol 2002;13: 2133-9.

17. Jaffer $Y$, Selby N, Taal M, Fluck R, McIntyre C. A meta-analysis of hemodialysis catheter locking solutions in the prevention of catheter-related infection. Am J Kidney Dis 2008; 51: 233-241.

18. Martínez Aranda MA, Arribas Cobo P et al. Eficacia del uso de bioconectores para los catéteres centrales de hemodiálisis. Rev Esp Enferm Nefrol 2011; 14 (2): 106-111.

19. Zafarghandi MR, Nazari I, Taghavi M, Salimi J, Moini M, Askarpour S. Comparison of results of placement of cuffed-tunneled hemodialysis catheter in internal jugular vein with subclavian vein for long-term dialysis. Pol Przegl Chir. 2013 Mar $1 ; 85(3): 133-6$. 